This item was submitted to Loughborough's Research Repository by the author.

Items in Figshare are protected by copyright, with all rights reserved, unless otherwise indicated.

\title{
Finding evidence in the dark: utilization of inkjet-printed amino acids
}

\section{PLEASE CITE THE PUBLISHED VERSION}

https://doi.org/10.1080/00450618.2019.1571108

\section{PUBLISHER}

(c) Australian Academy of Forensic Sciences. Published by Taylor \& Francis

\section{VERSION}

AM (Accepted Manuscript)

\section{PUBLISHER STATEMENT}

This is an Accepted Manuscript of an article published by Taylor \& Francis in Australian Journal of Forensic Sciences on 11 Feb 2019, available online: https://doi.org/10.1080/00450618.2019.1571108

\section{LICENCE}

CC BY-NC-ND 4.0

\section{REPOSITORY RECORD}

McMurchie, Beth, Paul Kelly, Roberto S.P. King, and George Torrens. 2019. "Finding Evidence in the Dark: Utilization of Inkjet-printed Amino Acids". figshare. https://hdl.handle.net/2134/37840. 


\title{
Finding Evidence in the Dark: Utilisation of Inkjet Printed Amino Acids Beth McMurchie ${ }^{\mathrm{a}, *}$, Paul Kelly ${ }^{\mathrm{a}}$, Roberto King ${ }^{\mathrm{b}}$, George Torrens ${ }^{\mathrm{a}}$ \\ ${ }^{a}$ Loughborough University, Epinal Way, Loughborough, Leicestershire, LE11 3TU, UK \\ ${ }^{b}$ Foster + Freeman Ltd, Vale Park, Evesham, Worcestershire WR11 1TD, UK
}

\begin{abstract}
.
Inkjet printing amino acids has been suggested as a method to obtain pseudo latent fingermarks which are identical to each other and can therefore be used to compare different fingermark development techniques. The following paper outlines how this method of printing amino acids was utilised to obtain standardised fluorescent patterns which could be used to assess individual's dark adaptation. Shapes, letters and patterns were printed in alanine using a standard inkjet printer, then developed using DFO to provide fluorescent images when viewed under green light and through a red filter. Images were also printed and developed using ninhydrin to obtain the resultant developed image in Ruhemann's purple. The use of the fluorescent patterns to assess dark adaptation lead to the confirmation that forensic examiners should dark adapt their eyes prior to looking for fluorescent evidence in the dark, as $16 \%$ more evidence was discoverable after waiting on average of 10 minutes in the dark prior to examination.
\end{abstract}

Keywords: inkjet printing; amino acid; dark adaptation

\section{Introduction}

When testing and comparing different latent fingermark development techniques, it can be difficult to determine whether differences observed are due to differences in the latent fingermark composition, rather than the quality of the fingermark development techniques. This can often lead to time consuming and participant heavy studies being performed in order to ascertain the quality and consistency of various fingermark development techniques. The use of inkjet printed amino acid based 'pseudo' fingermarks has previously been suggested as a method to obtain consistent and reliable fingermarks for initial testing of fingermark development techniques.[1] Since the initial use of inkjet printers to print artificial fingermarks, further research has been performed to improve the composition of amino acids and inorganic salts used to recreate the latent marks, alongside checking the reproducibility of printing the artificial marks.[2] In order to find the most evidence possible, forensic examiners are advised to dark adapt their eyes prior to looking for fluorescent evidence in the dark,[3] and allowing the eyes to dark adapt for only 10 minutes has shown to improve the amount of evidence discoverable by $16 \%$.[4] To obtain these results, the method of printing artificial fingermarks was utilised, providing a standardised fluorescent test. The method for testing inkjet printing of amino acids using a standard inkjet printer and developing the printed patterns using both DFO as a fluorescent reagent, and ninhydrin is explained below.

\section{Material \& Methods}

1,8-Diazafluoren-9-one (DFO) (WA Products, $0.1 \mathrm{~g}$ ) was added to a solution of petroleum ether (Sigma Aldrich, $156 \mathrm{ml}$ ), acetic acid (VWR, $4 \mathrm{ml}$ ), ethyl acetate (Fisher Scientific, $20 \mathrm{ml}$ ) and methanol (VWR, $20 \mathrm{ml}$ ) and stirred until all DFO had dissolved. Ninhydrin (Sigma Aldrich, $0.8 \mathrm{~g}$ ) was added to a solution of petroleum ether (Sigma Aldrich, $180 \mathrm{ml}$ ), ethyl acetate (Fisher Scientific, $14 \mathrm{ml}$ ), acetic acid (VWR, $2 \mathrm{ml}$ ) and methanol (VWR, $4 \mathrm{ml}$ ). Both a $0.4 \mathrm{mM}$ and $0.004 \mathrm{mM}$ solution of alanine were prepared in deionised water. An Epson Stylus Photo R265 inkjet printer was used throughout this study. Empty ink cartridges compatible with the Epson Stylus Photo R265 inkjet printer were purchased. The black ink cartridge was filled with the $0.4 \mathrm{mM}$ alanine solution. All samples were printed onto standard white copier paper. Letters in font type Arial were printed using the black ink cartridge ( $0.4 \mathrm{mM}$ alanine solution), in sizes $40-1$. These samples were developed via dipping of the sheets of paper in DFO solution, then heating in an oven at $100{ }^{\circ} \mathrm{C}$ for 20 minutes. The magenta ink cartridge was filled with $0.004 \mathrm{mM}$ alanine. A mixture of black and magenta shapes, letters and patterns were printed, and the sheets were developed via dipping of the paper sheets into DFO solution, then heating them to $100{ }^{\circ} \mathrm{C}$ in an oven for 20 minutes. A picture of Siegfried Ruhemann, the namesake of Ruhemann's purple was printed using the $0.4 \mathrm{mM}$ alanine solution. This sheet of paper was developed via dipping into ninhydrin solution, and heating in a humidified chamber in an oven at $100{ }^{\circ} \mathrm{C}$ for 20 minutes.

\section{Results}

The letters printed using the $0.4 \mathrm{mM}$ solution were viewed under green light excitation (490-560 nm) with an OG590 camera filter, and were legible down to size 5.From font size 4 onwards, the letters became too small and blurred to read, letters printed in font size 6-4 are shown in Figure 1. This shows that fine detail, such as that found in fingermarks can be obtained using a standard inkjet printer, with no alterations of special equipment.

The sheets containing shapes, letters and patterns printed using $0.4 \mathrm{mM}$ and $0.004 \mathrm{mM}$ alanine solutions and developed using DFO, provided a sheet of standardised fluorescent patterns which could be used to ascertain the amount of fluorescent evidence visible to participants during a study performed to assess the importance of dark adaptation. This work suggested that up to $16 \%$ more evidence could be located after dark adapting the eyes for as little as 10 minutes prior to looking for fluorescent evidence in the dark. [4] This shows that

* Corresponding author: b.mcmurchie@lboro.ac.uk 


\section{Page 2 of 2}

alongside providing standardised fingermarks for research purposes, inkjet printing of amino acids can be used to provide standardised fluorescent marks when using a fluorescent fingermark development reagent such as DFO, or 1,2-indanedione. The use of two different concentrations of alanine solution allowed there to be some shapes which appeared brighter and were therefore easier to visualise, than some of the shapes printed in the lower concentration of alanine, which appeared less intense and were therefore harder to visualise, particularly prior to dark adaptation. As inkjet printers usually contain at least 4 different coloured ink cartridges, black, cyan, magenta and yellow, a standard inkjet printer could provide 4 varied concentrations when printing amino acids. Similarly, different solutions of various amino acids, with the addition of inorganic salts could be printed to provide a variety of pseudo fingermarks.

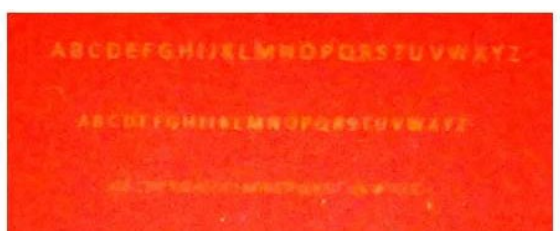

Figure 1 - Letters in Arial font sizes 6 - 4 printed in $0.4 \mathrm{mM}$ alanine and developed using DFO, shown photographed under green light excitation (490-560 nm) with an OG590 camera filter.

The sheets printed showing Ruhemann were developed using ninhydrin, and the resulting picture of Siegfried Ruhemann was visible under white light, due to the formation of Ruhemann's purple, the product formed from the reaction of amino acids with ninhydrin. This image is shown in Figure 2. This shows that alongside simple patterns, letters and shapes, complex and detailed patterns can be printed in amino acid solutions without a specialist printer and once developed with a suitable fingermark development reagent, are recognisable.

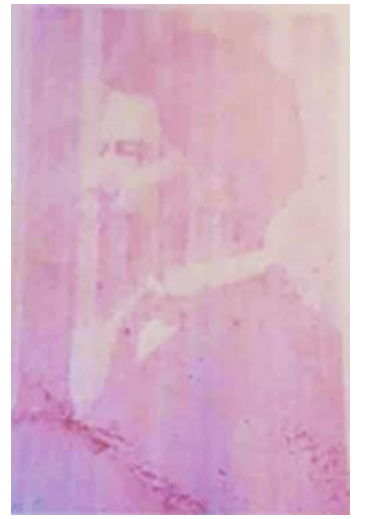

Figure 2 - An image of Siegfried Ruhemann printed in $0.4 \mathrm{mM}$ alanine and developed using ninhydrin.

\section{Conclusion}

The use of an inkjet printer has been shown to provide reliable and consistent prints of many shapes, letters, patterns and images in amino acids which can then be developed using standard fingermark development techniques. Alongside providing intricate pseudo fingermarks which can be used to test fingermark development reagents, they can be used to provide standardised fluorescent patterns for research, such as that performed assessing the effect of dark adaptation.

\section{Role of funding}

The PhD during which this research was undertaken was funded by Loughborough University; however this funding had no involvement in the study design, collection, analysis or publication of the data.

\section{Acknowledgements}

We would like to acknowledge Loughborough University, and the Royal Society of Chemistry, Analytical Division, who provided funding for the above research to be presented at ANZFSS 2018.

\section{Conflict of interest}

Conflict of interest: none.

\section{References}

[1] Schwarz L (2009) An amino acid model for latent fingerprints on porous surfaces. J Forensic Sci 54:1323-1326

[2] Hong S, Hong I, Han A, Seo JY, Namgung J (2015) A new method of artificial latent fingerprint creation using artificial sweat and inkjet printer. Forensic Sci Int 257:403-408

[3] Bandey HL, Bleay SM, Bowman VJ, Downham RP, G S V (2014) Fingermark Visualisation Manual.

[4] McMurchie B, King RSP, Kelly PF, Torrens GE (2018) The importance of dark adaptation for forensic examinations; an evaluation of the Crime-lite Eye ${ }^{\mathrm{TM}}$. Sci Justice. doi: 10.1016/j.scijus.2018.10.006 